\title{
Photometric CCD sequences for calibration of the ESO/SERC atlas ${ }^{\star \star \star \star}$
}

\author{
B. Cunow ${ }^{1,3}$, R. Duemmler ${ }^{2,3}$, G. Spiekermann ${ }^{3}$, R. Ungruhe ${ }^{3}$, and W.F. Wargau ${ }^{1, \dagger}$ \\ 1 Department of Mathematics, Applied Mathematics and Astronomy, University of South Africa, P.O. Box 392, Pretoria 0003, \\ Republic of South Africa \\ 2 Department of Geosciences and Astronomy, University of Oulu, Linnanmaa, P.O. Box 333, 90571 Oulu, Finland \\ 3 Astronomisches Institut der Westfälischen Wilhelms-Universität, Wilhelm-Klemm-Str. 10, 48149 Münster, Germany \\ $\dagger$ deceased in November 1996
}

Received October 31; accepted December 19, 1996

\begin{abstract}
For calibration of ESO/SERC survey plates, photometric CCD sequences have been obtained for 10 ESO/SERC fields in the area $10^{\mathrm{h}} 00^{\mathrm{m}}<$ R.A. $<15^{\mathrm{h}} 30^{\mathrm{m}}$ and $-34^{\circ}<$ Decl. $<-18^{\circ}$. The sequences contain $B, V$ and $R$ magnitudes for 117 galaxies and 162 stars with $12.7<V<21.0$.
\end{abstract}

Key words: techniques: photometric - galaxies: photometry — atlas

\section{Introduction}

In 1991, a project for obtaining CCD magnitudes of galaxies and stars with magnitudes fainter than $V=10$ was started at the Astronomical Institute of Münster University, Germany. This project is part of the cosmological Muenster Redshift Project MRSP (e.g. Seitter 1992; Schuecker 1996) which uses filmcopies of the $\mathrm{ESO} / \operatorname{SERC}(J)$ and $\operatorname{ESO} / \operatorname{SERC}(R)$ surveys and of lowdispersion objective prism plates obtained with the UK Schmidt Telescope.

The CCD sequences are used for magnitude calibration of the $J$ and $R$ plates. Their photometric passbands are defined by the IIIa-J emulsion and GG395 filter (wavelength region: $395 \mathrm{~nm}<\lambda<540 \mathrm{~nm}$ ) and by the IIIa-F emulsion and RG630 filter (wavelength region: $630 \mathrm{~nm}<\lambda<$

Send offprint requests to: B. Cunow

* Based on observations collected at the South African Astronomical Observatory, Sutherland, Republic of South Africa.

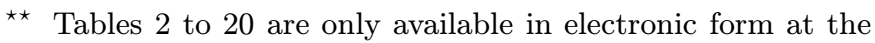
CDS via anonymous ftp to cdsarc.u-strasbg.fr (130.79.128.5) or via http://cdsweb.u-strasbg.fr/Abstract.html
$700 \mathrm{~nm})$, respectively. For calibration of the photographic magnitudes, $B, V$ and $R$ magnitudes are needed.

Since 1991, a large number of CCD sequences have been obtained for this project (Cunow 1993; Cunow \& Wargau 1993, 1994; Cunow \& Ungruhe 1995). These sequences cover $34 \mathrm{ESO} / \mathrm{SERC}$ fields in the area from $20^{\mathrm{h}} 30^{\mathrm{m}}$ to $5^{\mathrm{h}} 30^{\mathrm{m}}$ in right ascension and from $-73^{\circ}$ to $-20^{\circ}$ in declination. They contain $B, V$ and $R$ magnitudes for 131 galaxies and 190 stars, and $V$ and $R$ magnitudes for another 191 galaxies and 180 stars. Their magnitudes are in the range $11<V<22$. The CCD magnitudes are calibrated to the Kron-Cousins system.

In this paper, photometric CCD sequences of a different part of the sky are presented. They have been obtained in $10 \mathrm{ESO} / \mathrm{SERC}$ fields in the area $10^{\mathrm{h}} 00^{\mathrm{m}}<$ R.A. $<$ $15^{\mathrm{h}} 30^{\mathrm{m}}$ and $-34^{\circ}<$ Decl. $<-18^{\circ}$.

\section{Observations}

The CCD sequences have been observed in March 1995 with the $1.0 \mathrm{~m}$ telescope at the South African Astronomical Observatory (SAAO) in Sutherland, South Africa. The CCD camera contains a Tek chip of $512 \times 512$ pixels. The pixel size is $27 \mu \mathrm{m}$ which corresponds to a scale of $0.35 \mathrm{arcsec} /$ pixel and to an image size of $3{ }^{\prime} 0 \times 3{ }^{\prime} 0$. The filters available are Johnson $B$ and $V$ and Cousins $R$.

The CCD observations were made during five photometric nights. The seeing varied between $1^{\prime \prime} .5$ and 2 .'0 FWHM. In order to observe as many galaxies as possible, all sequences were taken in dense areas of clusters of galaxies chosen from Abell et al. (1989). For each ESO/SERC field (except field No. 576), two CCD fields were observed. For each CCD field $B, V$ and $R$ frames were taken. The exposure times were $1800 \mathrm{~s}$ in $B$ and $900 \mathrm{~s}$ in $V$ and $R$, respectively.

The CCD magnitudes are calibrated with the magnitudes of 18 E-region stars given by Menzies et al. (1980). 
The magnitude and colour ranges of the chosen stars were $8.13<V<10.65$ and $-0.02<B-V<1.53$, respectively. The standard stars were observed several times during a night at different airmasses with exposure times between $3 \mathrm{~s}$ and $12 \mathrm{~s}$ per frame. With these data, the coefficients for transforming the instrumental CCD system into the standard magnitude system were determined.

\section{Photometry}

The instrumental CCD magnitudes were measured using the procedures described in Cunow (1993). The dark current was found to be negligible for this CCD chip. The instrumental CCD magnitudes are aperture magnitudes with apertures being large enough to contain the whole object. The following aperture radii were used: 5 ". $3,6^{\prime \prime} 7$, 8 ". $1,99^{\prime \prime} 1,11^{\prime \prime} .6,13^{\prime \prime} 7,18^{\prime \prime} .2$ and $22^{\prime \prime} 8$. The aperture chosen for an individual object is the smallest one with a diameter which is at least twice as large as the object size. Checks by eye ensured that the chosen aperture is always larger than the whole object.


Fig. 1. Comparison of catalogue magnitudes with the CCD magnitudes for the E-region stars. a) shows the $B$ measurements, b) the $V$ measurements and c) the $R$ measurements

For transformation of the instrumental magnitudes to the standard system, the following equations were used:

$B_{\mathrm{CCD}}=B+k_{1, B}+k_{2, B} X+k_{3, B}(B-V)$
$V_{\mathrm{CCD}}=V+k_{1, V}+k_{2, V} X+k_{3, V}(B-V)$

$R_{\mathrm{CCD}}=R+k_{1, R}+k_{2, R} X+k_{3, R}(V-R)$.

$X$ is the airmass, $B_{\mathrm{CCD}}, V_{\mathrm{CCD}}$ and $R_{\mathrm{CCD}}$ are the instrumental magnitudes and $B, V$ and $R$ the magnitudes in the Kron-Cousins-System. The coefficients $k$ were obtained from the standard stars by a fitting procedure following the guidelines by Harris et al. (1981). Small colour terms were found:

$k_{3, B}=-0.066 \pm 0.008$

$k_{3, V}=0.017 \pm 0.010$

$k_{3, R}=-0.032 \pm 0.029$.

Table 1. List of cluster fields

\begin{tabular}{cccccc}
\hline Abell cluster & ESO/SERC & \multicolumn{2}{c}{$\alpha(2000.0)$} & \multicolumn{2}{c}{$\delta(2000.0)$} \\
no. & field no. & $\mathrm{h}$ & $\mathrm{m}$ & & ○ \\
\hline 0916 & 567 & 10 & 03.9 & -19 & 22 \\
$\mathrm{~S} 0645$ & 569 & 10 & 46.2 & -18 & 27 \\
3471 & 438 & 11 & 11.2 & -30 & 09 \\
3528 & 443 & 12 & 54.3 & -29 & 01 \\
$\mathrm{~S} 0726$ & 382 & 13 & 15.2 & -33 & 38 \\
1732 & 576 & 13 & 25.0 & -20 & 13 \\
3558 & 444 & 13 & 27.9 & -31 & 29 \\
3576 & 445 & 13 & 52.8 & -30 & 17 \\
1996 & 513 & 14 & 57.4 & -23 & 55 \\
3621 & 514 & 15 & 28.7 & -24 & 52 \\
\hline
\end{tabular}

Figure 1 shows the CCD magnitudes versus the catalogue magnitude for the E-region stars. From the scatter the random magnitude errors for these stars were determined for the different filters: $\sigma_{B}=0{ }^{\mathrm{m}} 031, \sigma_{V}=0{ }^{\mathrm{m}} 023$ and $\sigma_{R}=0$. 042. These errors are similar to the ones found for our previous CCD sequences (Cunow 1993; Cunow \& Wargau 1993, 1994; Cunow \& Ungruhe 1995). From this similarity and from the fact that in this work observations and reductions were done in the same way as for our previous CCD sequences, the same magnitude errors as given in those papers are expected for the programme objects in this work (from $\sigma=0{ }^{\mathrm{m}} 05$ for the bright objects to $\sigma=0$. 15 for the very faint objects).

\section{Catalogue of the CCD sequences}

CCD sequences have been measured in 10 southern Abell clusters. Table 1 gives the cluster coordinates according to Abell et al. (1989). The final catalogue, given in Tables 2 to 20, contains $B, V$ and $R$ magnitudes for 117 galaxies and 162 stars with $12.7<V<21.0$. Column 2 gives the object type, $\mathrm{S}$ for star, $\mathrm{G}$ for galaxy. The positions were measured from scans of the direct Schmidt plates with one of the PDS 2020 GM $^{\text {plus }}$ machines at the Astronomisches 
Institut, Münster, Germany. Their accuracy is between 0.5 and $1^{\prime \prime} .0$.

Acknowledgements. We thank Dr. R.S. Stobie for allocation of observing time at SAAO/Sutherland. We also thank the SAAO staff for assistance during the observations. It is a pleasure for GS to thank the Department of Mathematics, Applied Mathematics and Astronomy of the University of South Africa for the invitation, the financial support and for the hospitality during the stay at the University where a part of this publication was prepared. GS further thanks the Internationales Büro des Forschungszentrum Jülich and the BMBF for the financial support of the observing run under number S3539000. Special thanks go to Prof. W. Seitter and the other members of the Muenster Redshift Project (MRSP) for many useful discussions. Dr. A. Bruch kindly provided the programmes for magnitude transformation from the CCD system to the standard system. This work is part of the MRSP. Financial support of the MRSP by the DFG under numbers
Se 345/14-1,2,3 and Se 345/21-1 is gratefully acknowledged. Finally BC, RD, GS and RU thank Walter F. Wargau for a very fruitful and sucessful collaboration during the last years. Walter died shortly after this paper has been submitted. We will miss him.

\section{References}

Abell G.O., Corwin H.G., Olowin R.P., 1989, ApJS 70, 1

Cunow B., 1993, A\&AS 97, 541

Cunow B., Wargau W.F., 1993, A\&AS 102, 331

Cunow B., Wargau W.F., 1994, A\&AS 107, 277

Cunow B., Ungruhe R., 1995, A\&AS 112, 213

Harris W.E., Fitzgerald M.P., Reed B.C., 1981, PASP 93, 507

Menzies J.W., Banfield R.M., Laing J.D., 1980, Circ. S. Afr. Astron. Obs. 1, 149

Schuecker P., 1996, MNRAS 279, 1057

Seitter W.C., 1992, in Digitised Optical Sky Surveys, MacGillivray H.T. and Thomson E.B. (eds.). Kluwer, Dordrecht, p. 367 